\title{
The catalytic performance of metal-free defected carbon catalyst towards acetylene hydrochlorination revealed from first principle calculations
}

\author{
Sajjad Ali ${ }^{1}$, Muhammad Baber Khan ${ }^{2}$, Said Alam Khan ${ }^{3}$, and Noora $\mathrm{Ms}^{4}$ \\ ${ }^{1}$ Southern University of Science and Technology \\ ${ }^{2}$ government Post Graduate Jehanzeb College College \\ ${ }^{3}$ University of Malakand \\ ${ }^{4}$ Abdul Wali Khan University
}

May 29, 2020

\begin{abstract}
The defected carbon materials as a metal-free catalyst shown superior stability and catalytic performance in the acetylene hydrochlorination reaction. Through DFT calculation, for the first time several different defected configurations comprising mono, di-vacancies, and Stone Wales defect on single-walled carbon nanotubes (SWCNTs) have been used as a direct catalyst for acetylene hydrochlorination reaction. These defective sites on SWCNTs are the most active site for acetylene hydrochlorination reaction compared to pristine SWCNT. The different configuration of defects have different electronic structure, which specify that mono-vacancy defect, have more states adjecent to the Fermi level. The reactant acetylene (C2H2) adsorbed strongly than hydrogen chloride $(\mathrm{HCl})$ and expected to be the initial step of the reaction Acetylene adsorbed strongly at mono-vacancy defected SWCNT compared to other investigated defects. The reaction pathways analysis revealed that mono- and di-vacancy defected SWCNT has a minimum energy barriers and shows extraordinary performance towards acetylene hydrochlorination. This work suggests the potential of metal-free defected carbon to catalyze acetylene hydrochlorination and provide a solid base for future developments in acetylene hydrochlorination.
\end{abstract}

\section{Hosted file}

Manuscript.pdf available at https://authorea.com/users/327649/articles/455134-the-catalyticperformance-of-metal-free-defected-carbon-catalyst-towards-acetylene-hydrochlorinationrevealed-from-first-principle-calculations 